\title{
RECENT OBSERVATIONS ON THE MECHANICAL PROPERTIES OF POLYMER NETWORKS
}

\author{
P. THIRION and R. CHASSET \\ Institut Français du Caoutchouc. Paris, France
}

\begin{abstract}
The influence of several factors (temperature, elongation, swelling or dilution ratio, cross-link density, nature of the polymers and cross-linking agents) on the dynamic properties, creep and relaxation of polymer networks is surveyed in the terminal region of the spectrum.

Whereas the deformation does not change the relaxation kinetics in large ranges of extension, the cross-link density acts as a reduced variable apparently accelerating uniformly the viscoelastic processes beyond the glass transition. The other possible reductions 'time--temperature' and 'time-swelling' do not necessarily seem related to the variations of free volume.

From the view point of the explanation of the relaxation mechanisms in the terminal zone, the fact that the equilibrium of loosely cross-linked elastomers would only virtually be reached after several years at room temperature seem in better agreement with chain entanglement effects, either trapped or not by the permanent network, than with the dissociation of secondary linkages.
\end{abstract}

\section{INTRODUCTION}

$\Lambda$ series of recent studies originating mainly from the laboratory of Professor J. D. Ferry has disclosed that polymer networks display peculiar loss mechanisms at low frequencies, thus giving rise to a new interest for the study of dynamic or transient properties which previously did not seem essentially different from those found in the glass transition zone

Many obstacles, however, remain to be overcome in a field where experimental as well as theoretical difficulties abound. A primary one, for example. concerns the poor precision of measurements made near the equilibrium state, either owing to the decrease of the dynamic losses at low frequencies ${ }^{1}$, or because eventually aging always masks the residual viscoelasticity in the course of static tests, whatever precautions are taken ${ }^{2}$ On the other hand. the interpretation of transient phenomena requires a thorough knowledge of the networks structure. Yet, their topology seems far from being completely defined by a unique criterium: the cross-link density. the present methods of determination of which may be vitiated by the drawbacks of the statistical elasticity theory and too rough an assimilation of labile chains entanglements with chemical cross-links ${ }^{3}$.

In return, reduced variables methods widely used in other fields of polymer rheology ${ }^{4}$ have already provided, in particular for elastomer networks, much information on the kind of influence on the viscoelasticity of networks of such factors as temperature, deformation, swelling or dilution ratio and crosslink density. It is this broad approach which has been chosen here, in the 


\section{P. THIRION AND R. CHASSET}

hope of finding general laws which may provide a guide to further theoretical investigations.

\section{ISOTHERMAL TENSION-STRAIN-TIME RELATIONS}

The most precise way of analysing, independantly from temperature, the influence of the deformations amplitude on the vulcanisates viscoelasticity is to compare the stress diminution undergone as a function of time by test-pieces maintained at constant elongations. Beyond the glass transition zone, these stress relaxation curves are generally parallels on a logarithmic plot, which means that the stress can be resolved into a product of two terms, one depending on the deformation only and the other on time ${ }^{5.6}$. Also, to the extent where no structural modifications occur by crystallisation or chemical degradation, the transient component of stress tends to vanish asymptotically with time.

This factorisation principle, which has been successfully applied to mono or biaxial creep tests (increase of elongation at constant stress) and

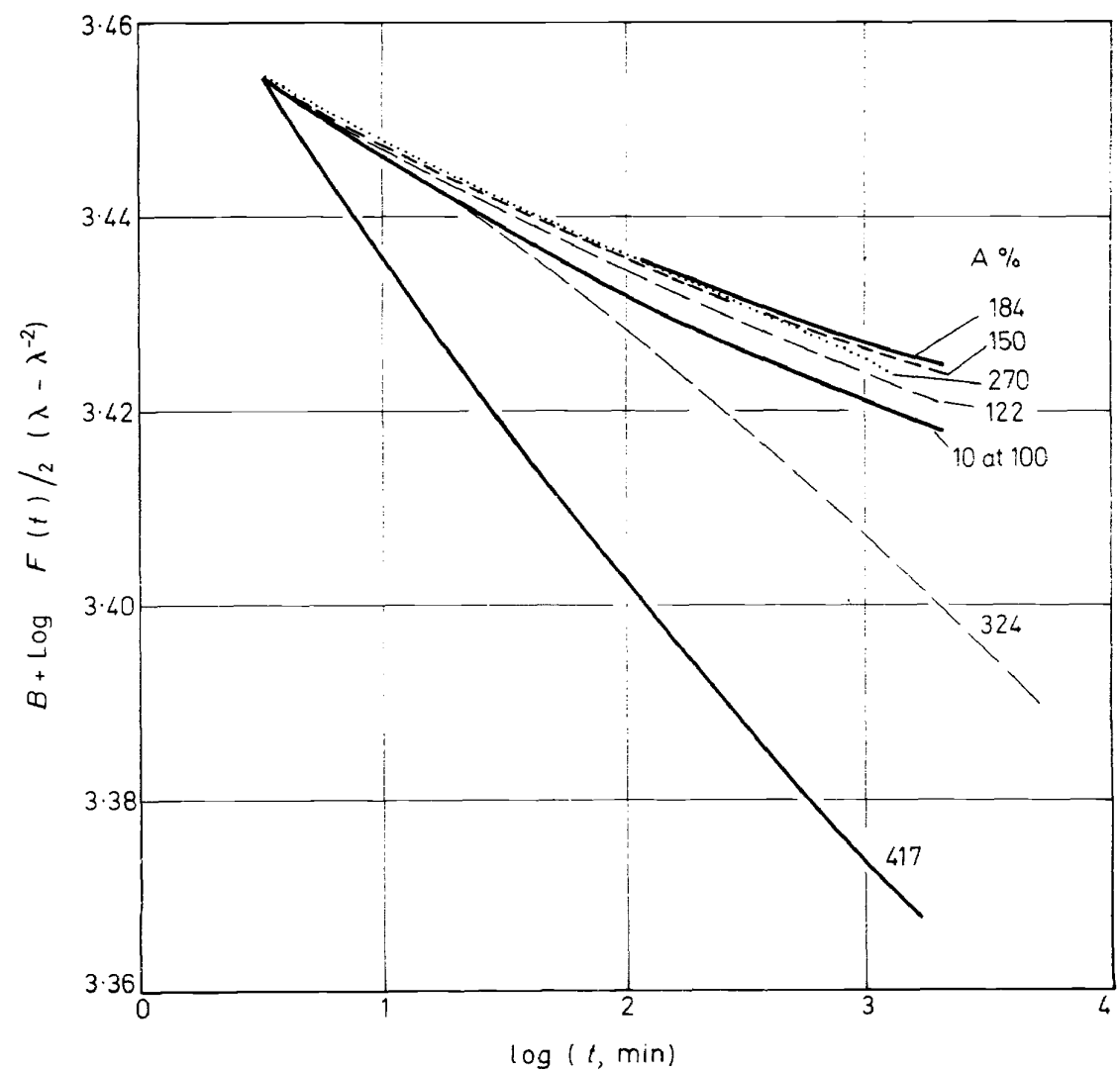

Figure 1. Influence of strain on the relaxation modulus of a natural rubber-dicumyl peroxide vulcanisate. Temperature $30^{\circ} \mathrm{C}$; the origin has been arbitrarily shifted so that the ordinates coincide at $3 \mathrm{~min}$. (Figure 2, ref. 11). 
to extension at constant speed ${ }^{7-10}$, also makes directly comparable the experimental data obtained either in the non linear domain of large deformations (as in the above cases), or in the linear domain of small deformations (as for example for dynamic sinusoidal shear).

At least from an analytical point of view, it is important to know, relative to possible experimental errors, the limits of validity of the factorisation principle. Figure 1 shows for a pure gum vulcanisate of natural rubberdicumyl peroxide - that these limits are not related to condensation of the polymer by crystallisation ${ }^{11}$. Until 100 per cent elongation, all the relaxation curves can be superimposed within $1 / 1000$, but the deviations observed relative to these kinetics are of opposite sense above or below about 300 per cent elongation. Moreover, in the case of cis-polybutadiene or of carbonblack filled vulcanisates, the factorisation applies equally well up to only 30 or 50 per cent elongation and the limits of validity of the principle must therefore depend largely on the nature of the systems considered.

The cause of the diminution of the relaxation above the preceding limits is not yet clear. At any rate, this effect cannot be explained by the isothermal expansion due to the extension, whereas the latter should play a dominant role near the glass transition zone as predicted by Ferry and Stratton by replacing in the WLF equation a temperature variable by a strain variable $e^{12}$. Because of the very shape of this homographic function, one should then observe a decreasing influence of strain when the latter increases, which is obviously not the case in the rubberlike zone.

Therefore, rather than free volume variations, much more specific orientation processes of the molecular chains probably intervene. In this respect, one may suppose according to a suggestion by Professor A. V. Tobolsky, that crystalline nuclei behave in the case of the above stereospecific polymers as covalent cross-links. But it is also possible that the stress factorisation itself results only from a fortuitious compensation of two different molecular processes and a drop of the relaxation spectrum should then be the rule beyond certain extensions, even for non-crystallisable polymers. Incidently, a similar modification of the spectrum has been reported for non-crosslinked polymers undergoing sinusoidal shears of large amplitude ${ }^{13}$.

\section{TIME-TEMPERATURE AND TIME-SWELLING RATIO REDUCTIONS}

In the glass transition zone, the rheological functions of time or frequency corresponding to various temperatures can be deduced from one another by means of the WLF equation, if the characteristic temperature $T_{\mathrm{s}}$ or the glass transition temperature $T_{\mathrm{g}}$ is known ${ }^{14}$. An homographic expression of the same form may be used to make a similar reduction by replacing temperature by the solvent dilution ratio ${ }^{12}$. In both cases, the free volume variation of the polymer causes a uniform modification of the relaxation times, which explains why the spectra undergo a mere time shift

The determination of master curves of the same type is still possible in the case of networks, in the rubber-like region, provided the modulus shift is not necessarily linked with the predictions of the statistical elasticity theory. In a reduction with respect to temperature, the corresponding 


\section{P. THIRION AND R. CHASSET}

modulus correction in fact becomes of the same order of magnitude as the stress variations as a function of time, which are much smaller than in the glass transition zone. Large errors may then arise from even a minute contribution of the internal energy or of unavoidable experimental errors. Moreover, the level of temperature considered, of the order of $160^{\circ} \mathrm{C}$ above the glass transition point, exceeds the normal limits of application of the WLF equation. Nevertheless, it has been found with natural rubber vulcanisates that the time--temperature shifts of creep curves are compatible with this equation ${ }^{15}$ and the latter has served to extrapolate numerous dynamic data obtained with various polymers, using, in certain cases, viscosity measurements in solutions ${ }^{16-20}$.

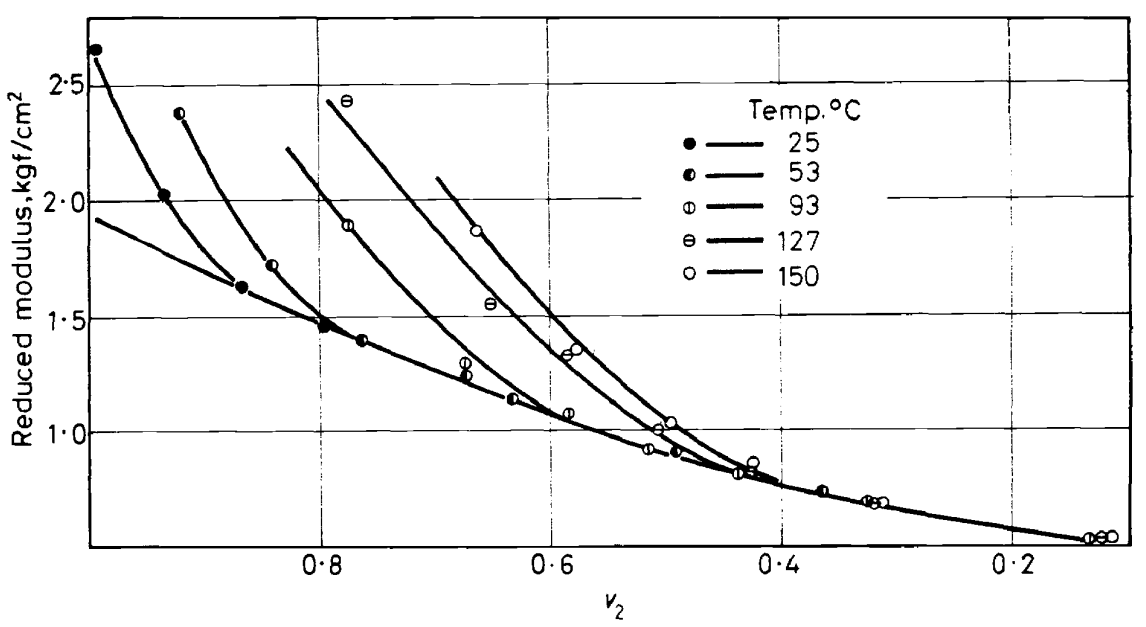

Figure 2. Variation of an isochronal modulus ( $300 \mathrm{~s}$ creep) reduced both with respect to temperature and to $v_{2} \frac{\frac{1}{3}}{3}$, as a function of the volume fraction $v_{2}$ of the polymer, for a network of 2-hydroxyethyl dimethacrylate metacrylate slightly cross-linked (Figure 1a, ref. 21).

More or less empirical shifts along the modulus axis are also required to allow a correct time- - swelling ratio reduction as shown, for example, by the creep measurements made after 5 minutes of extension between 0 and $150^{\circ} \mathrm{C}$ with hydrophilic networks directly formed during copolymerisation ${ }^{21}$. The samples studied have been prepared by the reaction of 2-hydroxyethylmethacrylate with ethylene dimethacrylate in the presence of water, then extracted and finally swollen with 2-2-oxydiethanol. Figure 2 represents, as a function of the volume fraction $v_{2}$ of the swollen polymer, the variations of the modulus reduced both with respect to temperature and swelling ratio, on the basis of the gaussian statistical theory. Instead of obtaining only one horizontal straight line for each network, in agreement with the theory, for this small degree of cross-linking, a series of isotherms has been found which merge with the equilibrium bottom envelope at swelling ratios which increase as the temperature is lowered.

The gaps respective to the envelope reflect transient effects which are normally very marked in poorly cross-linked systems. On the other hand, 


\section{MECHANICAL PROPERTIES OF POLYMER NETWORKS}

the slope of the equilibrium curve is always negative with swelling. This deviation of the gaussian statistical theory, which is directly related to the $\mathrm{C}_{2}$ term of stress-strain Mooney-Rivlin equation ${ }^{22}$, confirms the conclusions of other tests on polyurethane and rubber vulcanisates in the swollen state $^{23.24}$. In every case, the equilibrium modulus decreases more than by the $v_{2}^{\frac{1}{3}}$ coefficient indicated by the statistical theory. These deviations, the origin of which is rather uncertain, have recently been ascribed either to inter-molecular suprastructures ${ }^{23}$, or to various volume exclusion effects ${ }^{3,25}$ preventing, in particular, mutually entangled chains from crossing through each other.

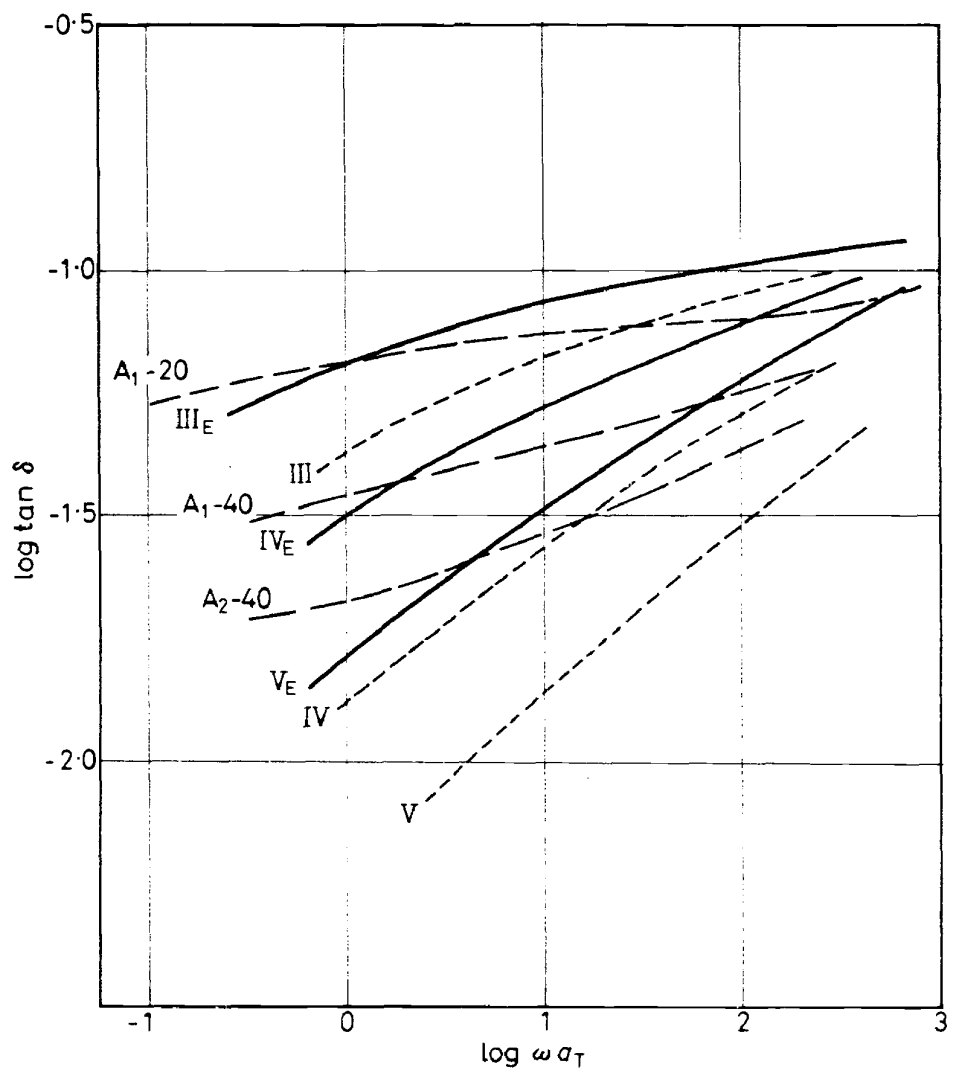

Figure 3. Variation of the loss tangent as a function of the reduced frequency, for natural rubber-dicumyl peroxide vulcanisates [ - networks formed in the swollen state with $37 \% \mathrm{ASTM}_{3}$ oil, then extracted; ..... same network formed in the swolleh state, not extracted; . . . . classical networks non-diluted (Figure 3, ref. 24)]

The transient properties should also be sensitive to the network topological peculiarities, and it is interesting to compare, from this view point, vulcanisates prepared in the dry or in the swollen state, particularly in view of the smaller number of trapped entanglements in the second case ${ }^{24}$. Figure 3 shows the behaviour of natural rubber- dicumyl peroxide vulcanisates, with nearly 
the same equilibrium modulus which have been prepared in the dry state, in the presence of 37 per cent oil and extracted afterwards (series $\operatorname{III}_{E}, \operatorname{IV}_{E}$, $\mathrm{V}_{\mathrm{E}}$ ). The $\mathrm{ASTM}_{3}$ oil used has a viscosity high enough, 33 centistokes, to avoid any evaporation during vulcanisation. The losses, characterized by $\tan \delta$ are in fact smaller at very low frequency for the extracted rubbers than for the other vulcanisates, and they increase in addition more rapidly as a function of frequency, in spite of the elimination of the solvent.

Moreover, the loss curves of the vulcanisates prepared in the swollen state, but not yet extracted (series III, IV, V), coincide with those of the extracted vulcanisates by a uniform shift of about one decade towards lower frequencies.

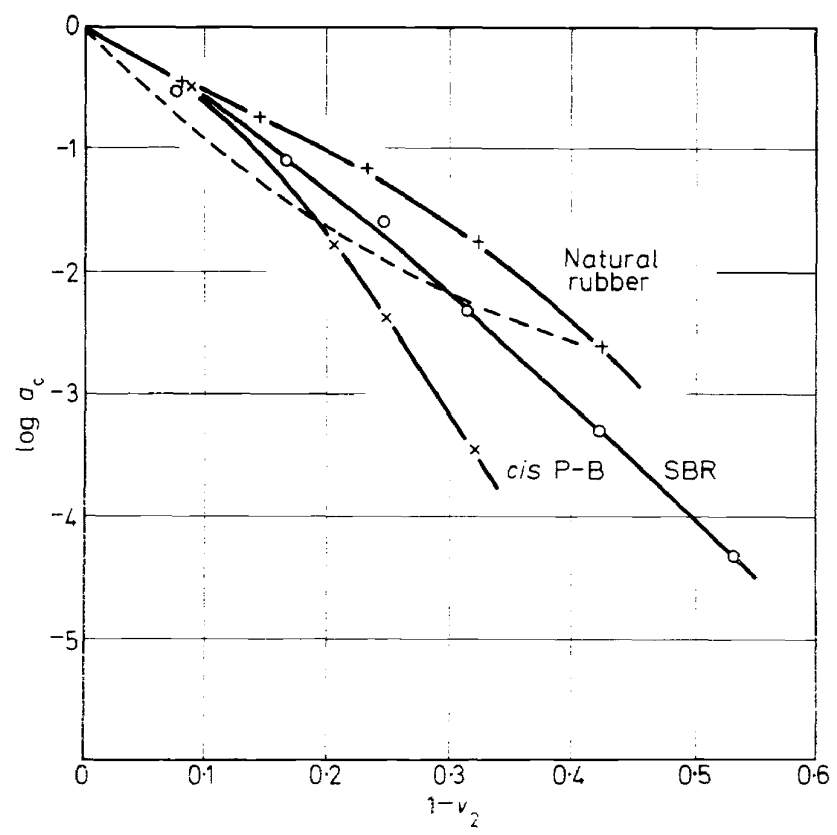

Figure 4. Variation of the shifting coefficients $a_{\mathrm{c}}$ as a function of the swelling ratio for three vulcanisates of natural rubber, cis-polybutadiene and SBR cross-linked by dicumyl peroxide [---- theoretical curve deduced from the free volume theory with arbitrary parameters] (ref. 26)

Furthermore, relaxation tests have also been made on natural rubber, SBR and cis-polybutadiene vulcanisates cross-linked in the dry state by dicumyl peroxide, then swollen with the above oil ${ }^{26}$. The curves corresponding to each swelling ratio all coincide with a master curve, and Figure 4 indicates the shifts as a function of the oil fraction. The dotted curve represents the variation which would be obtained if the homographic relation deduced from the free volume theory was valid, taking arbitrarily 0.15 for relative free volume of the solvent and 0.075 for that of the polymers. As the curvature is oriented in opposite senses, it does not seem, at least in this terminal zone of the spectrum, that the preceding analysis accounts for the effect of swelling on the vulcanisates viscoelasticity. 


\section{TIME-EQUILIBRIUM MODULUS REDUCTION}

The above reductions of variables may be envisaged for either linear polymers or networks. For the latter, one has to consider in addition the influence of the cross-link density on the viscoelastic properties. However, considering the unreliability of its present methods of determination ${ }^{3}$, it seems better to refer to directly measurable physical quantities, such as the modulus in the linear domain or, for more precision, to the stress $F_{\mathrm{e}}$ at equilibrium for a specified elongation, for example 50 per cent ${ }^{11}$.

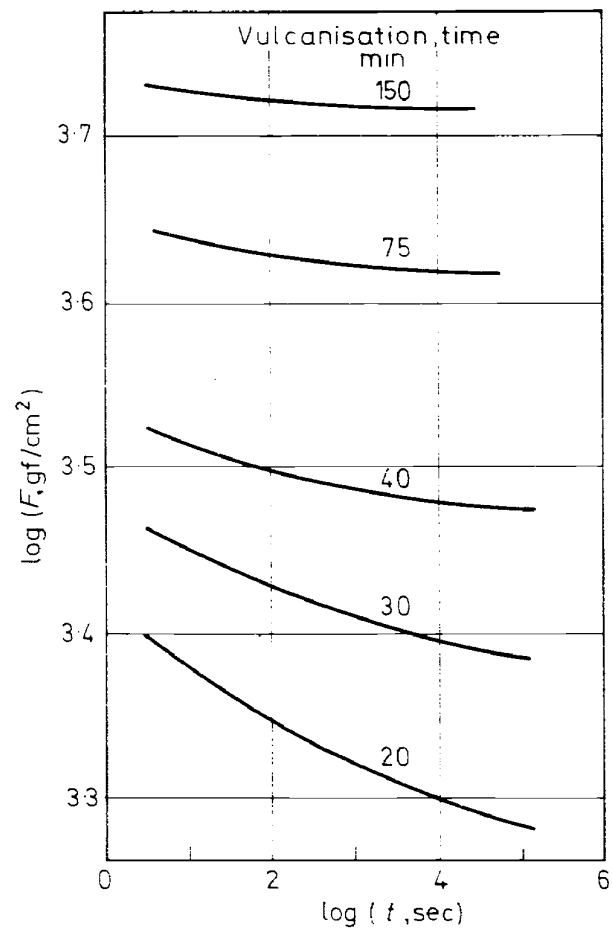

Figure 5. Influence of the vulcanisation time on the relaxation of a natural rubber-dicumyl peroxide mix. Temperature $30^{\circ} \mathrm{C}$. Elongation $50 \%$ (Figure 3, ref. 11)

Let us consider, then, a homogeneous series of vulcanisates prepared from the same polymer of high molecular weight by modifying either the amount of cross-linking agent, the temperature, or the curing time. One invariably discovers that, as soon as the glass transition is passed, their viscoelastic properties undergo considerable changes, essentially related to the evolution of the modulus and therefore to the cross-link density.

Figure 5 shows, for example, the diminution of amplitude of the relaxation observed at $30^{\circ} \mathrm{C}$ when the vulcanisation time at $135^{\circ} \mathrm{C}$ of a natural rubberdicumyl peroxide $(3.5 \%)$ increases from 20 to 150 minutes $^{6}$. Accordingly, a completely analogous behaviour has been found by Plazek by creep measurements on the same natural rubber samples, in spite of very different experimental conditions: shear smaller than 1 per cent using the WLF 


\section{P. THIRION AND R. CHASSET}

equation to extend the zone of the spectrum covered during isothermal tests between $1^{\circ}$ and $60^{\circ} \mathrm{C}^{15}$. One sees from Figure 6 that the shear compliance $J(t)$ varies with time in the same way as that derived from the preceding relaxation tests ${ }^{6}$, by means of a classical rheological transformation ${ }^{15}$; the level differences may be ascribed to errors of extrapolation and of dimensions measurement.

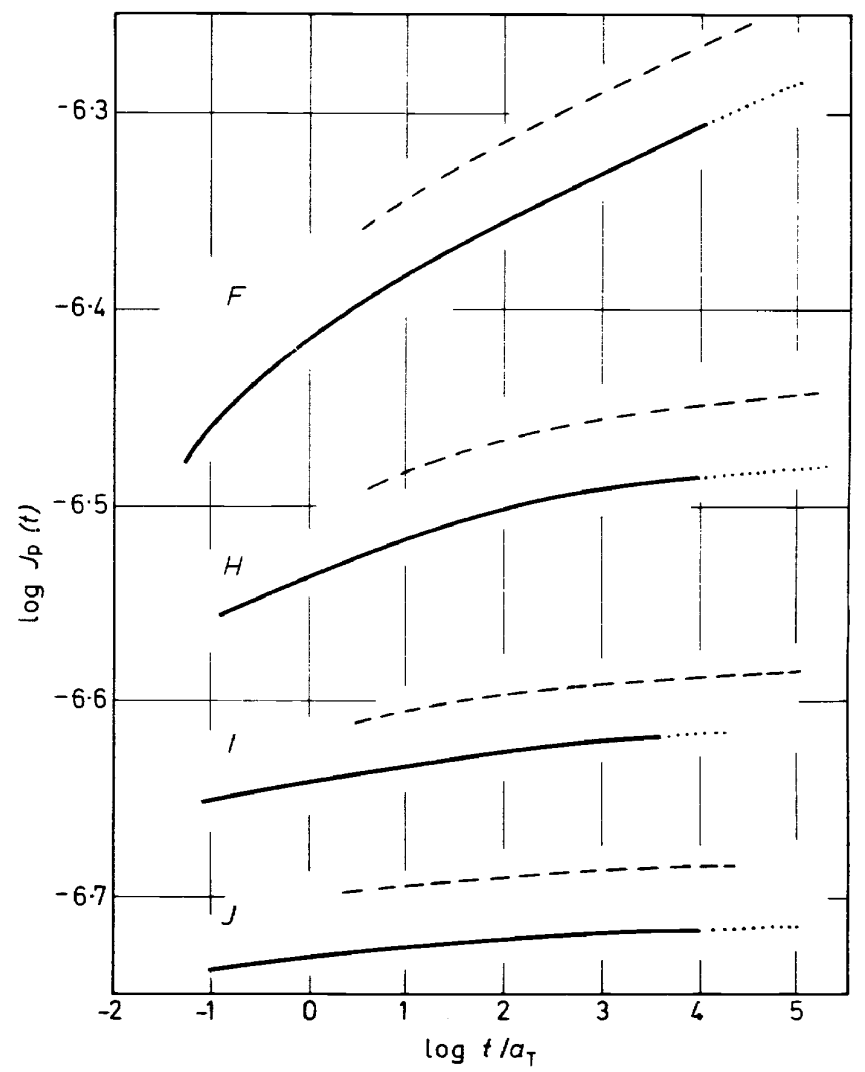

Figure 6. Variation of the dynamic compliance $J_{\mathrm{p}}(t)$, as a function of the reduced time for vulcanisates of natural rubber-dicumyl peroxide. Reference temperature $30^{\circ} \mathrm{C}$. [ - . - t transformation of the relaxation data of Figure 5 (Figure 2, ref. 15)]

The influence of the cross-link density on the dynamic properties $J^{\prime}, J^{\prime \prime}$ is also important when the frequency decreases. A slight modulus augmentation may correspond to a loss about ten times larger as appears, for example, on Figure 7 in the case of the above natural rubber samples, and even more spectacular effects have been observed on 40 per cent cis-53 per cent trans polybutadiene, just about the gel point (Figure 8$)^{17}$. The shape of the compliance curves $J^{\prime}(\omega), J^{\prime \prime}(\omega)$ does not, however, become immediately uniform when the influence of the glass transition stops. Indeed, in an intermediate range of frequency, a region appears where the losses tend to increase, 


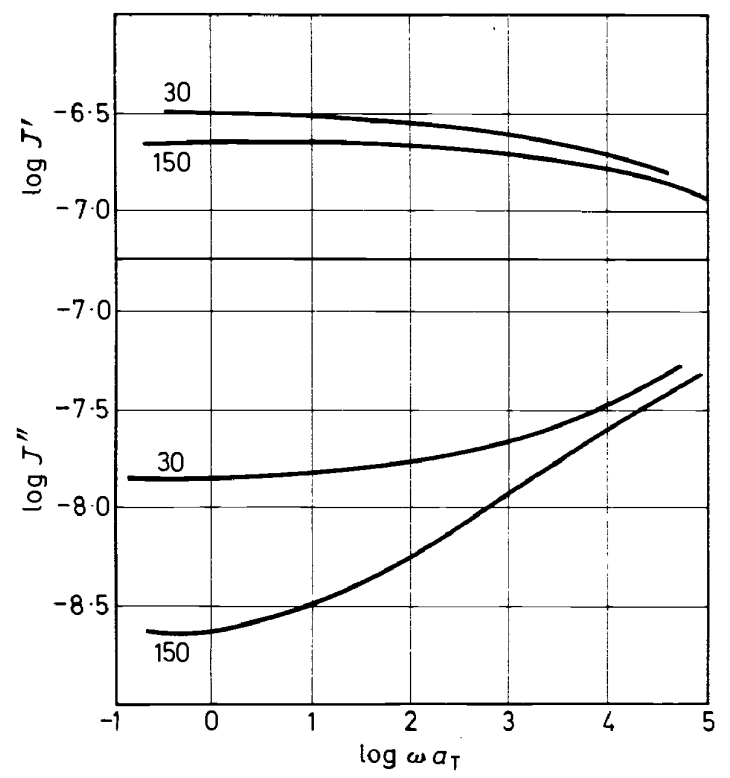

Figure 7. Components $J_{\mathrm{p}}^{\prime} J_{\mathrm{p}}^{\prime \prime}$ of the dynamic compliance reduced to $25^{\circ} \mathrm{C}$, as a function of the reduced frequency for two vulcanisates of natural rubber-dicumyl peroxide labelled by the vulcanisation time at $135^{\circ} \mathrm{C}$, in minutes (Figure 2, ref. 1)

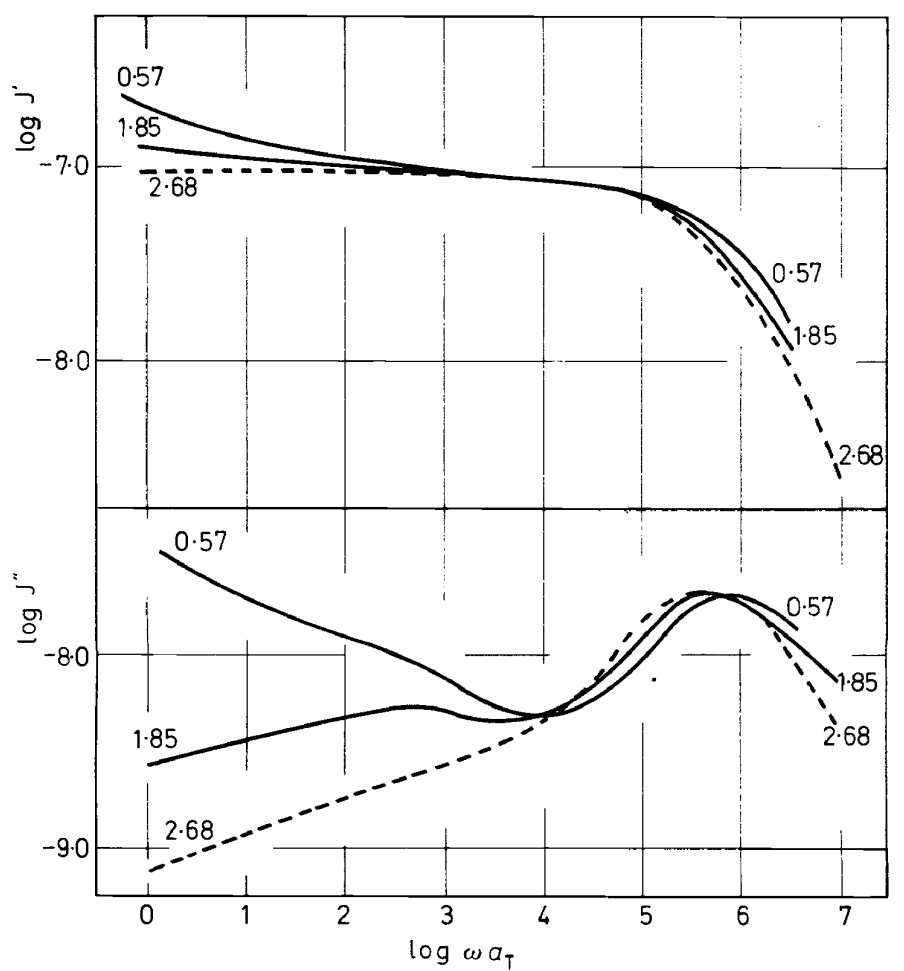

Figure 8. Components $J_{\mathrm{p}}^{\prime} J_{\mathrm{p}}^{\prime \prime}$ of the dynamic compliance reduced to $25^{\circ} \mathrm{C}$, as a function of the reduced frequency for three vulcanisates of $53 \%$ transpolybutadiene cross-linked by dicumyl peroxide and labelled by the cross-link density $\times 10^{4} \mathrm{moles} / \mathrm{cm}^{3}$ (Figure 2 , ref. 17) 
or at least to decrease less rapidly, when the frequency diminishes, and this feature is all the clearer as the cross link density is lower.

In the final part of the loss curves $J^{\prime \prime}(\omega)$, the diminution of the losses is, however, always monotonic, whereas in the same region of the spectrum the creep or relaxation kinetics may be closely approximated by a power function of time. In these conditions, it becomes possible to convert directly the data provided by one or the other of these three tests, by means of elementary Laplace transforms ${ }^{11}$.

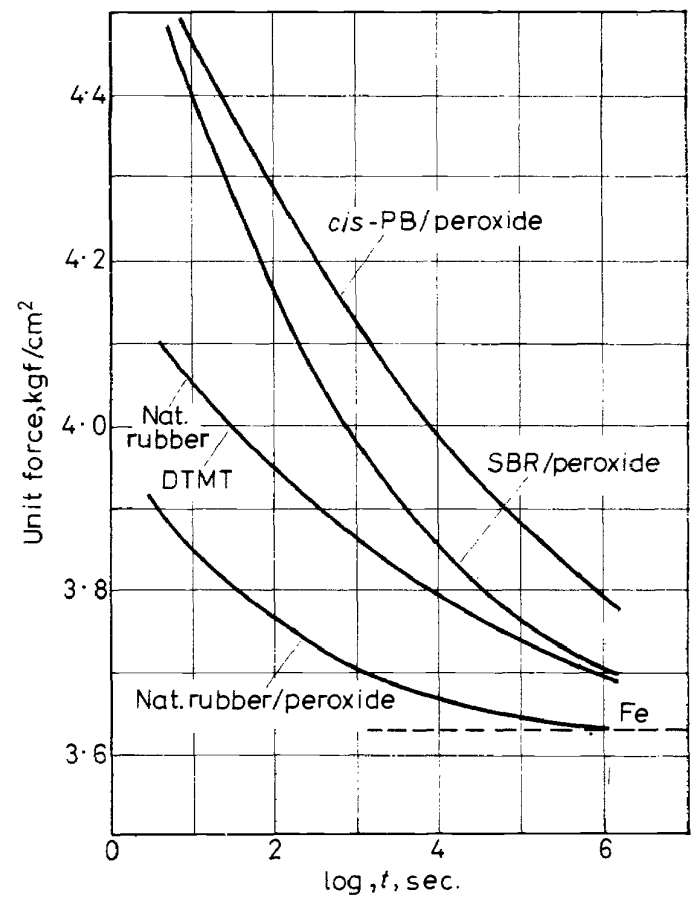

Figure 9. Relaxation of several elastomer vulcanisates with a same equilibrium modulus. Elongation $50 \%$. Temperature $30^{\circ} \mathrm{C}$ (Figure 10, ref. 11)

It is also in this terminal zone, which roughly coincides with that of most of the elastomers practical applications, that a new time-modulus reduction, peculiar to polymer networks, applies. Thanks to convenient shifts along the coordinates axes, the logarithmic curves of Figure 5 coincide in fact to within $1 / 5000$ with a unique master curve ${ }^{11}$, and this remarkable property has also been verified, either in creep or in relaxation, for several couples of polymers and cross-linking agents, so that it seems quite general (Figure 9).

In this way, therefore, one can determine at room temperature, without great risks of crystallisation or aging during tests, the creep or relaxation curve of even a slightly cross-linked vulcanisate, in a period of the order 
of 10 decades, which would normally be out of reach of direct experimentation. Knowing with respect to the chosen reference vulcanisate, the shifting coefficients $\log a_{v}$ along the time axis, one can easily deduce ${ }^{11}$ the viscoelastic properties of any other vulcanisate of the same type and of known modulus $F_{\mathrm{e}}($ Figure 10).

For example, this graphical extrapolation method indicates that it would be necessary to wait, in the virtual absence of aging, a year at $30^{\circ} \mathrm{C}$ to arrive at 5 per cent of the equilibrium in the case of the vulcanisate with a modulus of $2 \cdot 10^{6}$ dynes $/ \mathrm{cm}^{2}$, five hours for the vulcanisate with a double modulus and only 10 seconds for that with a modulus threefold larger. Consequently, only the densely cross-linked systems allow direet precise measurements of the elastic properties and many data of this kind reported in the literature are no doubt seriously in error owing to the extremely slow reactions of loosely cross-linked vulcanisates ${ }^{22}$

In addition to the stress-strain relations at equilibrium outside the linear domain, the relaxation master curves provide an objective characterization of the viscoelastic properties of each type of network, provided they are compared at equal modulus In this respect, it is a striking fact that neither

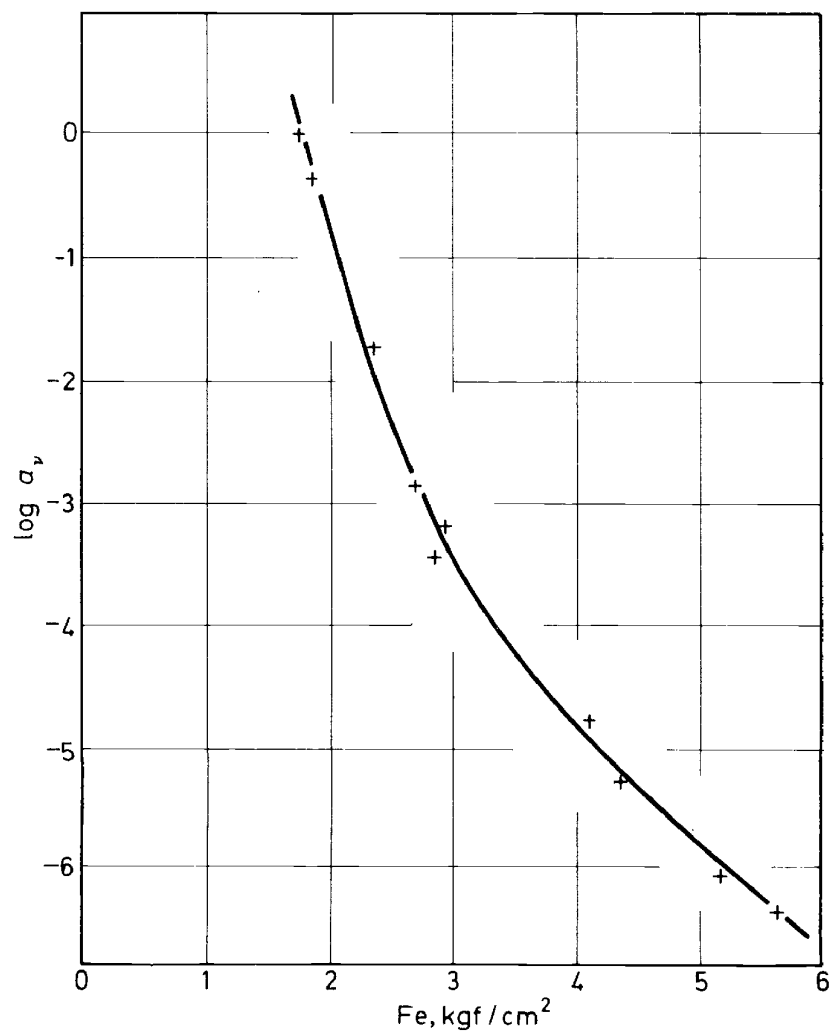

Figure 10. Variation of the shifting coefficient $a_{v}$ as a function of the equilibrium stress at $50 \%$ elongation. Natural rubber-dicumyl peroxide vulcanisate. Temperature $30^{\circ} \mathrm{C}$ (ref. 11) 
the nature of the polymer, nor that of the cross-linking agent are determined independently, the classification of the polymers according to their resilience being not necessarily in agreement with their glass transition temperature, in the terminal zone of the spectrum. So, all things equal, natural rubber cured by tetramethylthiurame disulphide tends to equilibrium more slowly than when cross-linked by dicumyl peroxide, and the couple cis-polybutadiene-dicumyl peroxide presents paradoxically, in these conditions, an even less ideally elastic behaviour than both the preceding types of vulcanisates ${ }^{11}$. In each of these cases, the shapes of the relaxation master curves are distinctly different (Figure 9).

On the contrary, these curves coincide exactly, thanks to a mere shift of about two decades along the time scale, for dicumyl peroxide vulcanisates of natural rubber and styrene 35-butadiene 65 copolymer (SBR), in spite of their very dissimilar chain microstructures. This strange contrast of behaviours reflects no doubt the complex topology of networks formed during the vulcanisation of elastomers. For instance, the radical chain reactions caused by dicumyl peroxide in cis-polybutadiene may entail a greater concentration of cross-links in certain regions than for polyisoprene, which may influence the speed of the molecular rearrangements following a deformation ${ }^{27}$.

\section{RELAXATION AND RETARDATION SPECTRA OF ELASTOMER VULCANISATES}

A combination of dynamic and static tests with the various possible reduction of variables allows, when applied to the same type of network, an analysis embracing periods of the order of 12 to 20 decades. The most general rheological representation is, then, the spectrum $H$ of the relaxation times, or the spectrum $L$ of the retardation times, the former emphasizing the short processes and the latter the slow ones ${ }^{14}$.

The relaxation spectra already disclose a nearly linear distribution, considerably flatter than that of non cross-linked polymers, this tendency being all the more marked as the cross-link density is smaller. In the case of the three dicumyl peroxide-butadiene styrene (SBR) vulcanisates, there appears in Figure 11 a transition region, occupying about three decades between the zone of convergency corresponding to the glass transition and the terminal zone of divergency. The alteration of the spectrum is, however, small and the corresponding transition still less marked, if not non-existing, for vulcanisates of dicumyl peroxide - natural rubber or cis-polybutadiene with the same modulus as the above SBR vulcanisates (Figure 12).

On the whole, the relaxation spectra of SBR and natural rubber both cured by this peroxide are strictly parallel throughout the terminal zone, whereas the spectrum of the cis-polybutadiene crosses those of the other two. The distribution of the relaxation times is indeed so spread out for the polybutadiene that, in this case, the final drop of the spectrum is not reached in the time limits explored. The beginning of this drop appears, however, clearly in the case of natural rubber or SBR, but with the limits of precision being obtained with the present equipment, one cannot say if the spectra tend towards an ultimate asymptote of finite or infinite slope. 
MECHANICAL PROPERTIES OF POLYMER NETWORKS

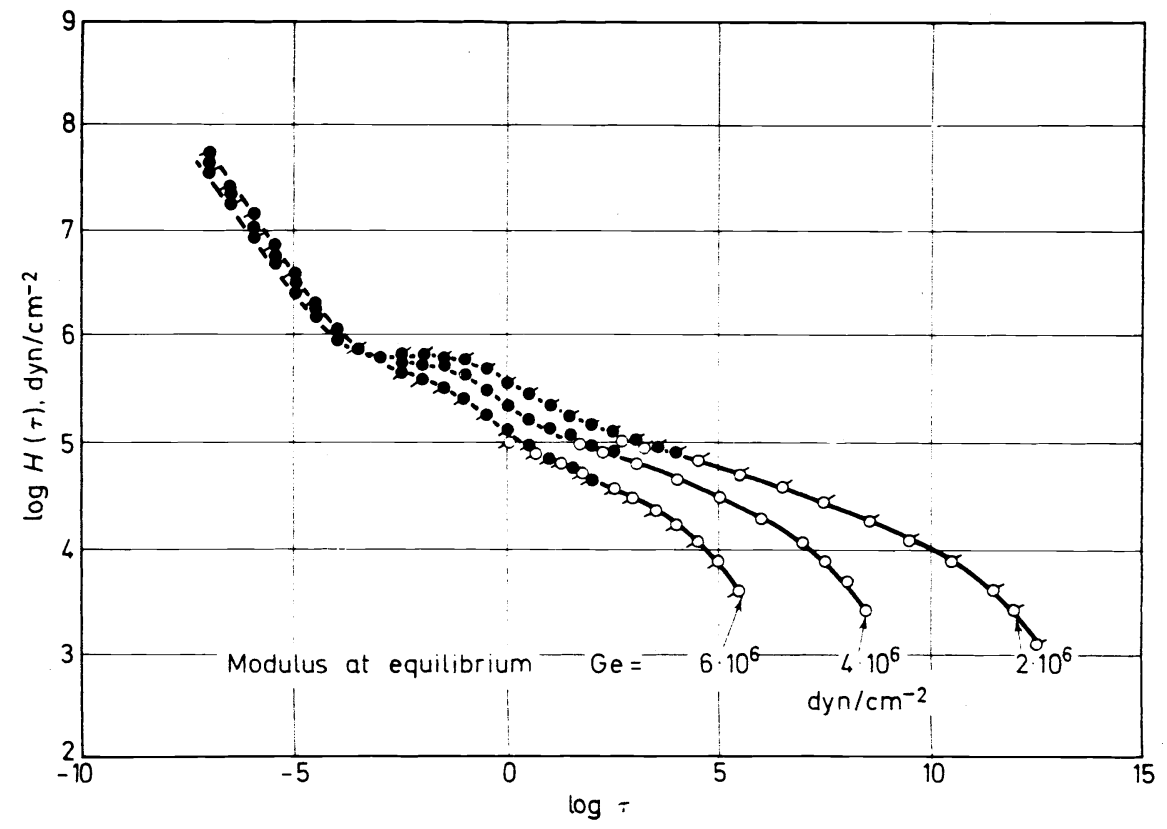

Figure 11. Influence of the cross link density on the distribution function of relaxation times for SBR vulcanised by dicumyl peroxide. Temperature $25^{\circ}$. [ $\bigcirc$ values deduced from the dynamic properties (Professor Ferry); $\cap$ values deduced from the relaxation properties (I.F.C.)] (ref. 26)

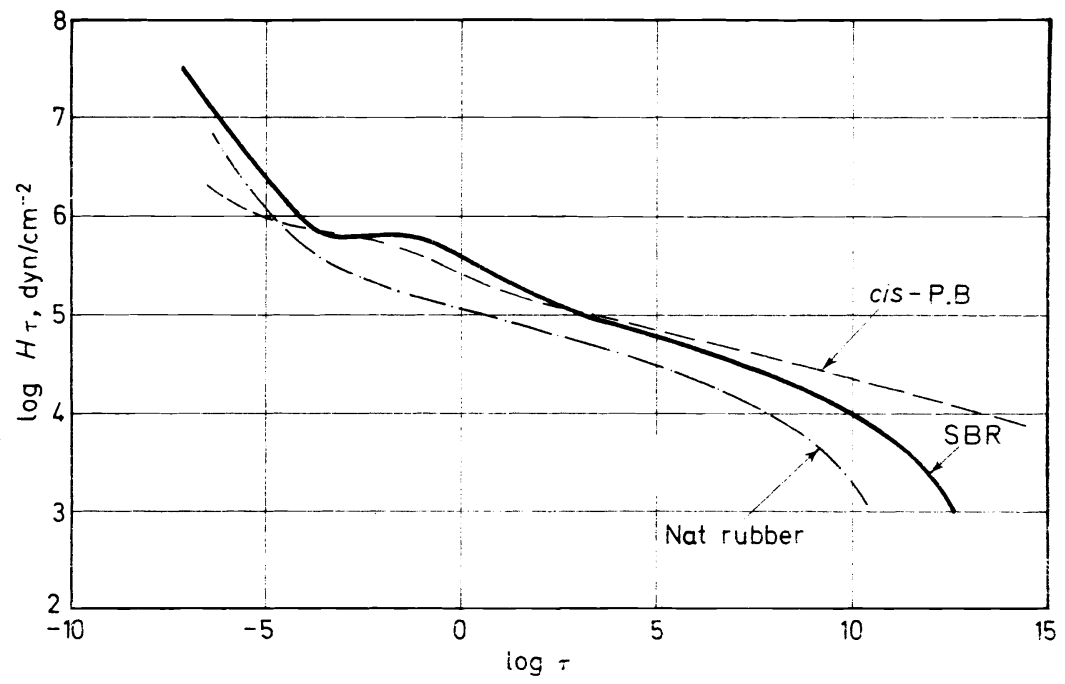

Figure 12. Relaxation spectra of natural rubber, SBR and cis-polybutadiene vulcanisates with same equilibrium modulus: $G_{\mathrm{e}}=2.10^{6} \mathrm{dyn} / \mathrm{cm}^{2}$. Temperature $25^{\circ} \mathrm{C}$ (unpublished work of Wisconsin University and I.F.C.) 


\section{P. THIRION AND R. CHASSET}

These conclusions are confirmed by the retardation spectra $L$ found either by Plazek ${ }^{15}$ with natural rubber, or by Ferry and co-workers in the case of 50 per cent trans-polybutadienes treated with sulphur (curves 1,2) or by dicumyl perioxide (curves 4 and 5 of Figure 13) ${ }^{28}$.

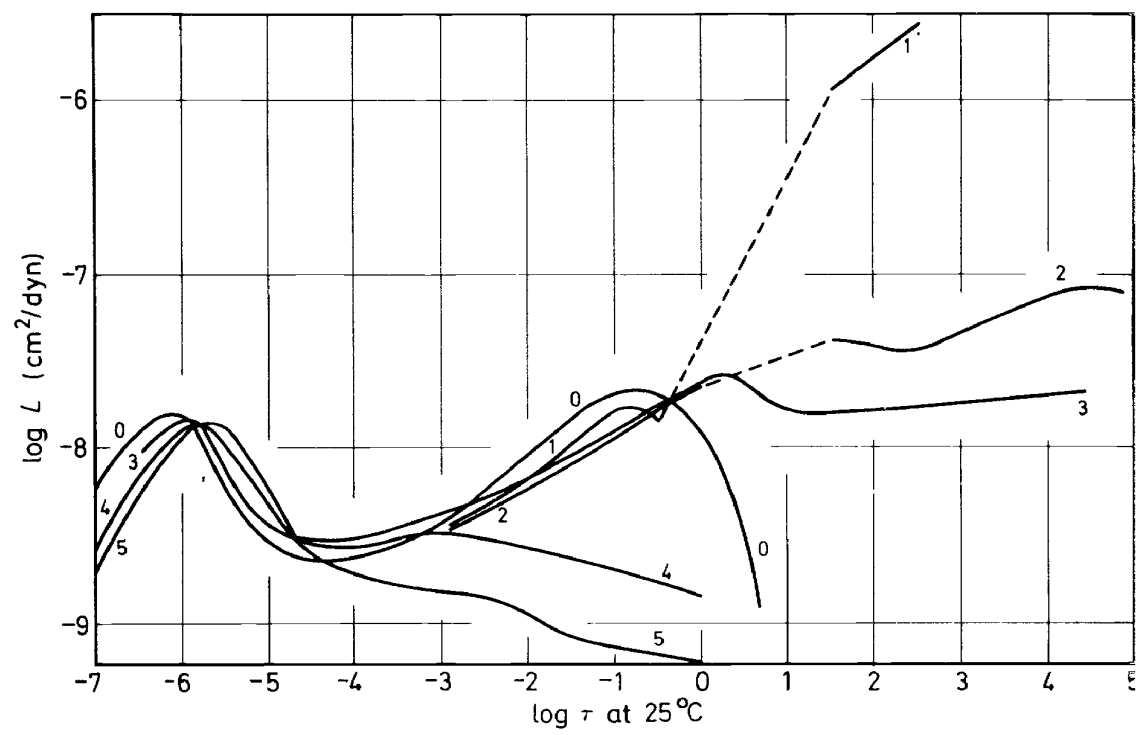

Figure 13. Retardation spectra of a $53 \%$ trans polybutadiene with several cross-link densities [0: precursor polymer non cross-linked (1) (2) (3): vulcanisation by a system sulphur-accelerator (4) (5): vulcanisation by dicumyl peroxide] (Figure 5, ref. 28)

Compared with the spectrum of a precursor polymer (curve 0), a strong increase of the final losses occurs in the neighbourhood of the gel point (curve 1). Then, as the cross-linking proceeds, the importance of this phenomenon regresses.

\section{MOLECULAR MECHANISMS CONTRIBUTING TO THE TERMINAL ZONE}

The most classical interpretation of the networks viscoelastic properties in the terminal zone of the spectrum is similar to that generally invoked for the 'rubberlike plateau' of linear polymers. One assumes that chain entanglements cause a coordination at long distance of non affine molecular rearrangements. The value of the monomeric friction coefficient remains, however, close to that of the precursor polymer, since the glass transition depends relatively little from the cross-link density ${ }^{14}$.

In the present case, however, two different kinds of entanglements should be distinguished. The first involves 'branches' or 'floating loops', more or less loosely fixed to the permanent network, and the mean spacing of such structures have been calculated by assimilating the low frequency viscoelastic behaviour of vulcanised rubbers with that of branched polymers not yet arrived at the gel point ${ }^{19,28}$. The second type of entanglement is formed by chains completely integrated to the permanent networks since they are 
directly linked to two, or better still to four, covalent cross-links. The latter trapped entanglements must contribute to the raising of the equilibrium modulus ${ }^{29}$, because they tend, as the cross-links themselves, to unfold the active molecular chains, But it is also possible that they promote energy losses during slow deformations ${ }^{15,30,33}$.

In such a theoretical frame, one conceives easily the qualitative effect of an increasing cross-linking on the viscoelastic properties of a polymer. As the mean length of the chains between successive cross-links approaches the mean entanglements distance, the longer elements are progressively eliminated from the transient network formed by the entanglements, either trapped or not. There must result a reduction of the relaxation mechanisms which should, however, preferentially affect the longest ${ }^{15}$.

This last remark raises a quantitative problem, since in reality the shape of the spectra does not at all depend on the cross-link density in the terminal zone. Even by taking into account the disordered character of the crosslinking, it is difficult to picture how the restrictive action of chemical crosslinks might lead to residual mechanisms of relaxation rigorously alike, although shifted in time, when the cross-linking density varies. The possible explanation, on the above basis of the absence of any modification of the relaxation kinetics or of the spectra, in large ranges of deformation, is not apparent, since a strong molecular orientation should apparently modify the entanglements.

Furthermore, it has been pointed out that the speed of creep and relaxation seems to depend on the nature of the polymer and the cross-linking agent. This remark hints at another physicochemical interpretation, based as the transient network theory of Yamamoto ${ }^{34}$ and Lodge ${ }^{35}$ on the dissociation of secondary linkages behaving as temporary junction points. For such a model, one may consider that the stress on the polymer in a quasi equilibrium state is, at each instant, the product of the thermal energy of all the 'active chain segments by a configuration term, function especially of the cross-link density and of the macroscopic deformation ${ }^{3}$.

From a mathematical view point, this decomposition of the stress. would naturally allow the introduction of the reductions of variables reported above, provided that the number of active segments (and therefore an energy term which is proportional) depends mainly on time only, the configuration term, on the other hand, being independant of time. The diminution of the stress during relaxation would then result from the release of floating elements of the network temporarily transformed in 'active' parts by the secondary linkages still linking them to the permanent network during the deformation. The relaxation kinetics would then be directly related to the dissociation probability of the seconciary linkages, itself ruled by the rate process theory ${ }^{36}$.

One of the drawbacks of this conception of the phenomenon is the importance of the gain in entropy of a chain so liberated from a deformed network, which should be approximately proportional to the square of the extension $^{37}$. As the speed of relaxation hardly depends on this last factor, one would have to admit that the heat of activation entirely compensates the entropy of activation, which does not seem a priori easily defensible.

Yet, the most serious objection concerns the considerable life time (in the order of years at room temperature) which should be attributed to a 
substantial part of the secondary linkages, a life superior indeed to that of certain covalent links in the same conditions. Quoting Professor Ferry, the theory seems, therefore, rather artificial, at least in the case of networks, as long as the nature of such junctions and the reason of their longevity are not known ${ }^{38}$. In this respect, recent studies on the reactivity of polyisoprenes and polybutadienes with oxygen point to the existence of sequences of conjugated double bonds along their molecular chains ${ }^{39-41}$. Furthermore, following a moderate oxidation of insaturated polymers, carbonyl and carboxyl groups may be formed, leading respectively to hydrogen or ionic bonds ${ }^{+2.43}$. The analogy with covalent cross-links indicates that in spite of their low concentration, the corresponding chain microstructures would still be able to influence the polymers' mechanical properties. The facts that these various groups form chemical weak points does not fit well, however with the long relaxation times experimentally found and entanglement effects appear, therefore, on the whole more credible than the secondary linkages hypothesis

\section{CONCLUSIONS}

More precise measurements than in the past have recently shown how sensitive the viscoelastic properties of vulcanisates are to the network topology, the cross-linking density playing a dominant role relative to the glass transition point of the polymer in the terminal zone of the spectrum. Consequently, a complex situation results of the diversity of the crosslinking physico-chemical processes, though certain reductions of variables make possible empirical mathematical simplifications, even in the case of large deformations.

The molecular mechanisms of relaxation, responsible for the flat distribution of relaxation and retardation times, seem qualitatively similar to those controlling the rubberlike plateau of linear polymers. But it would be interesting to distinguish the respective roles played by trapped entanglements and by entanglements formed by the pending chains of the precursor polymers, which may completely relax at equilibrium. It has already been found that the scission of the longest chains by means of a prolonged mastication slighly shifts the vulcanisates spectrum towards shorter times, at equal equilibrium modulus ${ }^{11}$. In this respect, it would be desirable to determine more rigorously the influence of the length of the precursor chains, by examining a series of vulcanisates prepared from monodisperse fractions of a same polymer.

Other experiments relating the structural chemical modifications with the rheological properties of the networks, or comparing completely saturated polymers and polydienes, would also allow an estimation of the eventual importance of intermolecular secondary linkages.

\section{ACKNOWLEDGEMENTS}

We sincerely thank Professor J. D. Ferry, Professor A. V. Tobolsky and Dr. D. Plazek for valuable discussions about this work. 


\section{References}

${ }^{1}$ J. D. Ferry, R. G. Mancke, E. Maekawa, Y. Oyanagi and R. Dickje, J. Phys. Chem. 68, 3413 (1964).

${ }^{2}$ P. Thirion and R. Chasset, Proc. 4th Rubber Technol. Conf. London, p. 338 (1962); Rev. Gén. Caout. Plast. 41, 271 (1964).

${ }^{3}$ P. Thirion and R. Chasset, Rev. Gén. Caout. Plast. 45, 859 (1968); Kautschuk u. Gummi 22, 281 (1969).

${ }^{4}$ M. L. Williams, R. F. Landel and J. D. Ferry, J. Am. Chem. Soc. 77, 3701 (1955).

${ }^{5}$ R. F. Landel and P. J. Stedry, J. Appl. Phys. 31, 1885 (1960).

${ }^{6}$ P. Thirion and R. Chasset, Proc. Int. Conf. Phys. of non crystalline solids. Delft (1964); Rubber Chem. Technol. 39, 870 (1966); Rev. Gén. Caout. Plast. 44, 1041 (1967).

${ }^{7}$ T. L. Smith, Trans. Soc. Rheol. 6, 61 (1962).

8 J. C. Halpin, J. Appl. Phys. 36, 2975 (1965).

9 J. A. C. Harwood and A. Schallamach, J. Appl. Polymer Sci. 11, 1835 (1967).

${ }^{10}$ T. L. Smith, Trans. Soc. Rheol. 12, 363 (1968).

${ }^{11}$ R. Chasset and P. Thirion, Proc. Nat. Rubber Conf. Kuala Lumpur (1968).

12 J. D. Ferry and R. A. Stratton, Kolloid Z. 171, 107 (1960).

${ }^{13}$ G. V. Vinogradov, Yu. Yanovsky and A. I. Isayev, communication Brit. Soc. Rheol. Glasgow, Sept. 1969.

14 J. D. Ferry, Viscoelastic Properties of Polymers. John Wiley and Sons, New York (1961).

15 D. J. Plazek, J. Polymer Sci. A2(4), 745 (1966).

${ }^{16}$ R. A. Dickie and J. D. Ferry, J. Phys. Chem. 70, 2594 (1966).

17 E. Maekawa, R. G. Mancke and J. D. Ferry, J. Phys. Chem. 69, 2811 (1965).

${ }^{18}$ R. G. Mancke and J. D. Ferry, Trans. Soc. Rheol. 12, 335 (1968).

${ }^{19}$ N. R. Langley and J. D. Ferry, Macromolecules 1, 353 (1968).

${ }^{20}$ J. F. Sanders, J. D. Ferry and R. H. Valentine, J. Polymer Sci. A2(6), 967 (1966).

21 J. Hasa and M. Ilavsky, Preprint no. 40 of Inst. Macromol. Chem., Prague (1968).

22 J. E. Mark and P. J. Flory, J. Appl. Phys. 37. 4635 (1966).

${ }^{23}$ R. Blockland and W. Prins, J. Polymer Sci. A2(7), 1595 (1969).

${ }^{24}$ N. R. Langley, R. A. Dickie, Chu-Ping Wong, J. D. Ferry, R. Chasset and P. Thirion, $J$. Polymer Sci. A2(6), 1371 (1968).

${ }^{25}$ L. S. Priss, Dokl. Acad. Nauk. SSSR 116, 225 (1957).

${ }^{26} \mathrm{R}$. Chasset, unpublished work.

27 B. M. E. Van der Hoff, Appl. Polym. Symp. 7, 21 (1968).

${ }^{28}$ R. H. Valentine, J. D. Ferry, T. Homma and K. Ninomiya, J. Polymer Sci. A2(6), 479 (1968).

${ }^{29}$ N. R. Langley. Macromolecules 1, 348 (1968).

${ }^{30}$ A. J. Chompff and J. A. Duiser. J. Chem. Phys. 45, 1505 (1966).

${ }^{31}$ A. J. Chompff and W. Prins. J. Chem. Phys. 48, 235 (1968).

${ }^{32}$ F. Bueche. Physical Properties of Polymers. Interscience Publ., New York (1962).

${ }^{33}$ J. C. Halpin. J. Appl. Phys. 36, 2975 (1965).

${ }^{34}$ M. Yamamoto. J. Phys. Soc. Japan 11, 413 (1956).

35 A. S. Lodge. Elastic Liquids. Academic Press, New York (1964).

${ }^{36}$ T. G. Fox, S. Gratch and S. Loshaek. in Rheology, F. K. Eirich, Academic Press, New York (1956).

${ }^{37}$ L. R. G. Treloar. The Physics of Rubber Elasticity. 2nd ed., Clarendon Press, Oxford (1958).

${ }^{38}$ J. D. Ferry. Private communication.

${ }^{39}$ F. J. Linnig, E. J. Parks and J. E. Stewart. J. Res. Nat. Bur. Std. A, 68, 499 (1964).

${ }^{40}$ T. Colglough, J. I. Cunneen and G. M. C. Higgins. J. Appl. Polymer Sci. 12, 295 (1968).

41 J. Morand. Rev. Gén. Caout. Plast. 45, 615 (1968).

42 B. C. Sekhar. Proc. Nat. Rubber Conf. Kuala Lumpur, p. 512 (1960).

${ }^{43}$ B. C. Sekhar, Proc. 4th Rubber Technol. Conf. London, p. 460 (1962). 